\title{
R3D2: Relativistic Reactive Riemann problem solver for Deflagrations and Detonations
}

\section{DOI: $10.21105 /$ joss. 00016}

\section{Software}

- Review ¿

- Repository ca

- Archive ¿

\section{Licence}

Authors of JOSS papers retain copyright and release the work under a Creative Commons Attribution 4.0 International License (CC-BY).

\section{Alice Harpole ${ }^{1}$ and lan Hawke ${ }^{1}$}

1 University of Southampton

\section{Summary}

This code extends standard exact solutions of the relativistic Riemann Problem to include a reaction term. It builds on existing solutions for the inert relativistic Riemann problem, as described by (Martí and Müller 2015), and of the non-relativistic reactive Riemann problem, as given by (Zhang and Zheng 1989).

Models of ideal hydrodynamics, where there is no viscosity or dissipation, can have solutions with discontinuities such as shocks. A simple case is the Riemann Problem, where two constant states are separated by a barrier. After the barrier is removed the solution develops, with waves (such as shocks and rarefactions) separating constant states.

The Riemann Problem has three main uses. Efficient, often approximate, solvers are an integral part of many modern hydrodynamic evolution codes. Second, the exact solution is a standard test for such codes. Finally, the solver can illustrate features of discontinuous solutions in more complex scenarios.

In Newtonian hydrodynamics, the Riemann problem is one-dimensional: the solution depends only on the normal component of any vector quantities in the initial conditions. However, in relativistic systems, the Lorentz factor introduces a coupling between the normal and tangential components. As found by (Rezzolla and Zanotti 2002), for high enough tangential velocities, the solution will smoothly transition from one wave pattern to another while maintaining the initial states otherwise unmodified. This code allows such transitions to be investigated in both inert and reactive systems.

\section{References}

Martí, José Maria, and Ewald Müller. 2015. "Grid-based Methods in Relativistic Hydrodynamics and Magnetohydrodynamics." Living Reviews in Computational Astrophysics 1. doi:10.1007/lrca-2015-3.

Rezzolla, Luciano, and Olindo Zanotti. 2002. "New Relativistic Effects in the Dynamics of Nonlinear Hydrodynamical Waves." Physical Review Letters 89 (11): 114501. doi:10.1103/PhysRevLett.89.114501.

Zhang, Tong, and Yuxi Zheng. 1989. "Riemann problem for gasdynamic combustion." Journal of Differential Equations 77 (2): 203-30. doi:10.1016/0022-0396(89)90142-3. 